\title{
Authentication Graphs: Analyzing User Behavior Within an Enterprise Network
}

\author{
Alexander D. Kent* \\ Los Alamos National Laboratory, Los Alamos, NM \\ Lorie M. Liebrock \\ New Mexico Institute of Mining and Technology, Socorro, NM \\ Joshua C. Neil \\ Los Alamos National Laboratory, Los Alamos, NM
}

\begin{abstract}
User authentication over the network builds a foundation of trust within largescale computer networks. The collection of this network authentication activity provides valuable insight into user behavior within an enterprise network. Representing this authentication data as a set of user-specific graphs and graph features, including time-constrained attributes, enables novel and comprehensive analysis opportunities. We show graph-based approaches to user classification and intrusion detection with practical results. We also show a method for assessing network authentication trust risk and cyber attack mitigation within an enterprise network using bipartite authentication graphs. We demonstrate the value of these graph-based approaches on a real-world authentication data set collected from an enterprise network.
\end{abstract}

Keywords: user authentication, network authentication, graph analysis, intrusion detection, cyber security, enterprise security

\section{Introduction}

User authentication is a fundamental aspect of modern computer use. Authentication enables computer systems to recognize specific users through a shared secret or other jointly recognized means. This authentication can take the form of a simple username and secret password or involve more complex means of identity involving varying factors (e.g., biometrics). Within enterprise

\footnotetext{
* Corresponding author

Email addresses: alex@lanl.gov (Alexander D. Kent), liebrock@cs.nmt.edu (Lorie M. Liebrock), jneil@lanl.gov (Joshua C. Neil)
}

Preprint submitted to Elsevier

September 1, 2014

(C) 2014. This manuscript version is made available under the Elsevier user license http://www.elsevier.com/open-access/userlicense/1.0/ 
computer environments, users often authenticate to many computer systems and applications throughout the network. In this environment, authentication activities are often provided by a unified network authentication mechanism. This network authentication capability and its encompassing centralized account management is a mainstay of modern information technology (IT) administration; without it, significant challenges would exist to manage accounts and authentication credentials across large numbers of individual systems (Kent and Clifford, 2000). Centralized management and authentication is considered both a best practice and expectation in most large organizations' IT infrastructure (Johnson et al., 2010).

Because network authentication events are generated for many activities, including access to applications and information of importance to an organization, these events provide great insights into the behavior of the authenticating users. In addition, because this user authentication activity implies directional relationships between computers within an enterprise network, they can be easily and efficiently represented as directed graphs or digraphs as defined by BangJensen and Gutin (2009). We find that these user authentication graphs provide a useful representation, enabling a platform for behavioral analytics based on a variety of induced graph attributes.

Note that the focus of this work is on improving the integrated security of all computers within an organization's network. We assume that enterprise cyber defense exists as an overlapping set of approaches that includes the network interior and a distributed set of collected data that includes user authentication activity. Given this assumption, which is the basis of how Los Alamos National Laboratory (LANL) conducts cyber defense, the work presented here is about analyzing the significant and aggregate user authentication activity across a large population of users and computers.

This paper is organized as follows: Section 2 presents our real-world authentication data set and foundation for analysis. Section 3 then formally presents our graph constructs and graph attributes used for analyzing authentication activity and behavior. We then discuss the application of our graph-based approach in Section 4 for both user classification and user compromise detection. Next, we present an alternate bipartite graph representation of authentication events, useful for network-wide risk analysis, in Section 5. Finally, related work, future work, and conclusions are discussed in Sections 6, 7, and 8, respectively.

\subsection{Key Contributions}

This paper makes the following novel research contributions:

- Formally define user authentication graphs as a representation of user authentication activity and behavior.

- Time-constrained graph features relevant to user authentication activity.

- A graph feature approach to user classification.

- A graph feature approach to intrusion detection. 
- A method for assessing network authentication trust risk and cyber attack mitigation within an enterprise network using bipartite authentication graphs.

\section{Real-World, Enterprise-Scale Data Set}

As the basis of exploration and analysis for this work, we utilized a comprehensive authentication data set from the enterprise network at LANL. This data comes from two sources: the event logs from centralized authentication servers (Microsoft Active Directory servers) and the cumulative event logs from individual networked computers running the Microsoft Windows operating system (desktops and servers). These relevant authentication events originate from both Kerberos (Neuman and Ts'o, 1994) and Microsoft's NTLM (Glass, 2006) authentication activity contained within the event logs.

This data set spans one calendar year of contiguous activity spanning 2012 and 2013. It originated from 33.9 billion raw event logs (1.4 terabytes compressed) collected across the LANL enterprise network of approximately 24,000 computers. Processing this raw data into normalized authentication event activity was done using the FileMap map/reduce system (Fisk, 2014) and required 26.1 weeks of serial processing time but was completed in 10.4 hours across a $320 \mathrm{CPU}$ (1 terabyte of aggregated memory) compute cluster. Normalization requirements included text processing from the non-well-formed authentication log events (variable field matching), matching relevant authentication activity from the wide variety of information within the disparate logs, and normalizing IP addresses, computer names, and user names.

We removed all authentication events to the Active Directory servers because every user that is part of the domain continuously authenticates to the domain controllers for various automated housekeeping purposes. We also removed all computer-account activity (user names ending with the special "\$" designation) and only consider human-driven and associated system authentication events. Finally, the computer-specific accounts LOCAL, SYSTEM, ANONYMOUS, and ADMINISTRATOR were also remove from the data since they are not relevant for enterprise-level integration. The resulting one year data set encompasses 11,593 users using 23,331 computers and accounts for 1,077,544,803 authentication events (a 6.3 gigabyte compressed data set). Figure 1 shows two example raw log events and the resulting directed graph that is induced.

In addition to the general population of 11,593 users within the data set, we also consider two subgroup populations useful in showing the value of authentication graphs. The first is a group of 439 users who have been determined to have various levels of privileged administrative access (root, administrator, etc) within the network. These administrative users were determined based on administrative group membership within the Active Directory domain. Examination of the administrative users and general users groups was conducted using the full one year data set and is detailed in Section 4.1.

The second group is a set of 81 users who were compromised during a monthlong red team exercise within the LANL enterprise network. This event sim- 
ulated a real malicious attack within the network. Because this attack was contained within one month's data, we have used this reduced time frame for analysis relating to the compromise activity to avoid relevant graph feature dilution. In the one month relevant to these compromise events, 85,668,332 authentication events occurred representing a typical month of authentication activity within the larger data set. The compromised user group is detailed in Section 4.2 .

The graph representing all user authentication activity within the one year data set has 23,331 vertices (corresponding to the number of computers) and 256,898 arcs (directed edges) representing network authentication activity. This integrated graph has a diameter of 19 and a time-constrained diameter of 17 (see Section 3). The size and complexity of this graph representing all authentication behavior within the network provides an appreciation for the overall connectedness and general complexity of an enterprise network. It is examined as a bipartite graph of users and computers in Section 5. In addition, we see opportunities for future research on this large, complex graph form. However, we have focused on the examination of individual user authentication subgraphs within Sections 3 and 4 . That is, we examine just the authentication activity for each individual user across the time period of the data set (one year for general and administrative users, 1 month for compromised users). This data generates 11,593 unique directed subgraphs, one for each user.

\section{Authentication Subgraphs}

These directed graphs representing each user's authentication activity over a time period we refer to as a Person's Authentication Subgraph (PAS). Figure 2 shows a typical PAS representing a single user's authentication activity across the one year data set discussed in Section 2.

Differentiating and exploring the attributes of these individual PASes yields useful results. We find that most users have a very static set of systems they authenticate to over time, reflecting how static most users' authentication activity is within the network, as seen with the representative user in Figure 2. Figure 3 shows the histogram of the number of vertices a PAS may have and also shows that administrative users generally have larger, more complex graphs compared to general users as seen within the tail. This increased complexity of administrative users can been seen in Figure 4 and is discussed in Section 4.1.

More specifically, we define a PAS as the directed subgraph $G_{u}\left(V_{u}, A_{u}\right)$ for user $u$ representing the user's authentication activity over some predefined time period (one year or one month within this paper). The vertex set $V_{u}$ is defined as the computers that user $u$ has one or more authentication events. The arc set $A_{u}$ represents the authentication activity from one computer to another for user $u$ within the network. ${ }^{1}$ We represent these arcs individually as $(x, y)$, indicating

\footnotetext{
${ }^{1}$ In terms of graph terminology within this paper we refer to arcs as directed edges within a directed graph and to edges as non-directional edges within an undirected graph.
} 
the directed edge from vertex $x$ to vertex $y$. An arc may represent more than one authentication event in cases where there are multiple authentication events from $x$ to $y$ within the time period of the PAS. A complete list of variables and associated descriptions can be found in Table 1.

We define a path as a sequence of arcs from vertex $v_{1}$ to vertex $v_{n}$ in $G_{u}$ :

$$
\begin{aligned}
P_{u}\left(v_{1}, v_{n}\right)= & \left\langle\left(v_{1}, v_{2}\right), \ldots,\left(v_{i-1}, v_{i}\right),\left(v_{i}, v_{i+1}\right), \ldots,\left(v_{n-1}, v_{n}\right)\right\rangle \\
& \text { s.t. }\left(v_{j}, v_{j+1}\right) \in A_{u}, j=1, \ldots, n-1 .
\end{aligned}
$$

Note that for each arc in a path, the head vertex of the arc must be the same as the tail vertex of the previous arc. Additionally, this definition does not preclude a path containing one arc.

The path length, $|P(x, y)|$, is the number of arcs within a path $(n-1$ in the above definition). PASes may have cycles within them $(P(v, v))$, but do not have self-cycles (an arc directly connecting a vertex to itself, $(v, v)$ ). In cases where there is a local authentication event on $v$, we instead ensure $v$ exists within the PAS. PASes may also have multiple connected components and as a result are not necessarily strongly connected, thus $P(x, y)$ and/or $P(y, x)$ may be undefined.

The path distance $d(x, y)$ is the shortest directed path length $|P(x, y)|$ from vertex $x$ to vertex $y$. We define the diameter $D_{u}$ to be the maximum distance $d(\cdot, \cdot)$ between any two vertices within $G_{u}$ :

$$
D_{u}=\max d(x, y) \text { s.t. } x, y \in V_{u} \wedge P_{u}(x, y) \neq \emptyset .
$$

Time constraint within path exploration provides additional value to our graph attributes. This is a form of graph constraint satisfaction (Kumar, 1992) within a PAS. To take advantage of time constraints, we define two time attributes that are assigned to every arc $(x, y)$ within a PAS. We define $t_{f i r s t}(x, y)$ to be the first time the arc $(x, y)$ was observed within the data set defining $G_{u}$. Similarly, the last time the arc $(x, y)$ was observed within the data set defining $G_{u}$ is denoted $t_{\text {last }}(x, y)$. When there is exactly one authentication event presented by an arc in the PAS, $t_{f i r s t}(x, y)=t_{\text {last }}(x, y)$. Keeping two time attributes per arc instead of every time instance reduces memory usage for PASes where large numbers of authentication events may be represented by an arc.

By requiring time ordering along the arcs in a path, the number of paths requiring investigation can be reduced. As such, we define a time constrained path from $v_{1}$ to $v_{n}$ in $G_{u}$ as:

$$
\begin{aligned}
\overrightarrow{P_{u}}\left(v_{1}, v_{n}\right)= & \left\langle\left(v_{1}, v_{2}\right), \ldots,\left(v_{i-1}, v_{i}\right),\left(v_{i}, v_{i+1}\right), \ldots,\left(v_{n-1}, v_{n}\right)\right\rangle \text { s.t. } \\
& t_{\text {last }}\left(v_{j}, v_{j+1}\right) \geq \max \left(t_{\text {first }}\left(v_{1}, v_{2}\right), \ldots, t_{\text {first }}\left(v_{j}, v_{j+1}\right)\right), \\
& \text { where }\left(v_{j}, v_{j+1}\right) \in A_{u}, j=1, \ldots, n-1 .
\end{aligned}
$$

The constraint of $t_{\text {last }}$ following all preceding $t_{\text {first }}$ values within a path correctly reflects event time ordering that exists in a sequential set of authentication events. Figure 5 demonstrates the difference of a time-constrained path within 
a small example graph versus a (longer) non-time-constrained path within the same graph.

A time-constrained path distance $\vec{d}(x, y)$, similar to its previously seen traditional definition, is the shortest directed path length $|\vec{P}(x, y)|$ from vertex $x$ to vertex $y$ using time-constrained arcs. We define a time-constrained diameter $\vec{D}_{u}$ to be the maximum time-constrained distance $\vec{d}(\cdot, \cdot)$ between any two vertices within $G_{u}$ :

$$
\vec{D}_{u}=\max \vec{d}(x, y) \text { s.t. } x, y \in V_{u} \wedge \vec{P}_{u}(x, y) \neq \emptyset .
$$

We find that time-constrained diameter is generally lower than non-timeconstrained diameter of the same PAS, particularly for users that have high diameters within our data set, as seen in Figure 6. It is possible for timeconstrained diameters to be longer than the non-time-constrained diameter (e.g., the PAS in Figure 12), though such examples are rare within the data set. Time-constrained paths and diameter provide graph features that improve the detection of malicious activity and the ability to differentiate user types (see Figures 13 and 14). Time-constrained paths also enable PAS simplification for visualization purposes, particularly for those that have a significant number of vertices and arcs. For example, Figure 9 shows a more complex PAS that has been decomposed into two time-constrained subgraphs based on a different starting vertex in each case. This simplification and visualization of the graphs with time enforcement could help cyber intrusion investigators better understand how potential compromises have occurred through authentication credential attacks.

In addition to graph paths, we also consider the directed graph density to be an important attribute describing how well connected a PAS is. We define the directed graph density $\rho_{u}$ as the ratio of arcs to potential arcs given the number of vertices:

$$
\rho_{u}=\frac{\left|A_{u}\right|}{\left|V_{u}\right|\left(\left|V_{u}\right|-1\right)} \text {. }
$$

Figure 7 shows the distribution of graph density across the population of PASes. We see that while administrative users often have larger PASes, they generally have lower density. The intuition is that administrative users make elongated graphs, reducing density.

The number of weakly connected subgraphs of $G_{u}$ that are not connected to each other is another relevant PAS attribute. A weakly connected subgraph is defined as a set of vertices where all associated arcs have directionality removed (becoming non-directional edges) such that there exists a non-directional path $P(x, y)$ for all vertices $x$ and $y$ within the weakly connected subgraph. We refer to this set of weakly connected subgraphs or connected components within $G_{u}$ as $C_{u}$. Figure 8 shows the distribution of connected component counts, $\left|C_{u}\right|$, for users' PASes within our authentication data sets. We find that the number of weakly connected subgraphs often corresponds well to the number of desktop computers a user uses (desktops, laptops, terminal servers, and similar); we also 
believe the count is skewed higher at LANL due to the common use of terminal services for certain business applications by many users.

Each vertex $v \in V_{u}$ has an indegree denoted $\operatorname{deg}^{-}(v)$ representing the number of arcs that arrive at $v$ (incoming authentication events to $v$ ) and an outdegree denoted $\operatorname{deg}^{+}(v)$ representing the number of arcs that originate from $v$ (outgoing authentication events).

The remainder of definitions in this section are all relative to the vertices within a PAS.

We define the set of vertices $O_{u}$ as outstars where each member vertex has an outdegree greater than 1 and outdegree is greater than or equal to indegree:

$$
O_{u}=\left\{v \in V_{u} \mid \operatorname{deg}^{+}(v)>1 \wedge \operatorname{deg}^{+}(v) \geq \operatorname{deg}^{-}(v)\right\} .
$$

Generally, we find that desktop computers and similar computers from which users originate activity are outstars. Similarly, we define the set of vertices $I_{u}$ as instars where each member vertex has indegree greater than 1 and indegree is greater than outdegree: ${ }^{2}$

$$
I_{u}=\left\{v \in V_{u} \mid \operatorname{deg}^{-}(v)>1 \wedge \operatorname{deg}^{-}(v)>\operatorname{deg}^{+}(v)\right\} .
$$

Network servers are generally classified as instars within a PAS.

Vertices that have both an indegree and outdegree of zero (no arcs associated with it) are contained within the set of isolated vertices $Z_{u}$ :

$$
Z_{u}=\left\{v \in V_{u} \mid \operatorname{deg}^{-}(v)=0 \wedge \operatorname{deg}^{+}(v)=0\right\} .
$$

Isolated vertices are generally computers where local authentication activity occurs but with no associated network authentication activity.

Returning to time constraints, we define the set of time-constrained transit vertices $\vec{T}_{u}$ where each member vertex $v$ has at least one arc proceeding from a parent vertex and one arc succeeding to a child vertex. In addition, all of the arcs from proceeding parent vertices to $v$ must have $t_{\text {first }}$ values that are before or at the same time as all of the $t_{\text {last }}$ values for arcs to succeeding child vertices from $v$ :

$$
\begin{aligned}
\vec{T}_{u}= & \left\{v \in V_{u} \mid \operatorname{deg}^{-}(v) \geq 1 \wedge \operatorname{deg}^{+}(v) \geq 1\right. \text { s.t. } \\
& \forall x(x, v) \in A_{u} \wedge \forall y(v, y) \in A_{u}, \\
& \left.t_{\text {first }}(x, v) \leq t_{\text {last }}(v, y)\right\}
\end{aligned}
$$

Figure 10 demostrates a transit vertex within a small example graph. Transit vertices are often associated with terminal servers or similar systems where users are authenticating first to and then from the computer.

Pseudo-leaf vertices account for the remainder of vertices that are not already contained in one of the previously defined outstar, instar, isolated, or transit

\footnotetext{
${ }^{2}$ Traditional graph definitions would call our outstars sources and similarly call our instars sinks. We believe our names are more representative of their nature within PASes.
} 
vertex sets. We define a set of pseudo-leaf vertices $L_{u}$ where both indegree and outdegree are 1 and 0,0 and 1 , or 1 and 1 , respectively:

$$
\begin{aligned}
L_{u}=\left\{v \in V_{u} \mid\right. & \left(\operatorname{deg}^{-}(v)=1 \wedge \operatorname{deg}^{+}(v) \leq 1\right) \\
& \left.\vee\left(\operatorname{deg}^{+}(v)=1 \wedge \operatorname{deg}^{-}(v) \leq 1\right)\right\} .
\end{aligned}
$$

Pseudo-leaf vertices within a PAS are generally incidental to its overall composition, but most often represent destination servers when indegree is one and outdegree is zero. Note that our definition of a pseudo-leaf vertex is somewhat more generalized compared to the usual definition for a leaf vertex, since it may have both indegree and outdegree. However, within our time-constrained definitions, these multi-degree pseudo-leaf vertices are similar to traditional leaf vertices within more specific time frames since they do not meet the previous definition as transit vertices.

Given the situation where a vertex could be a member of $O_{u}, I_{u}$, or $L_{u}$ as well as $\vec{T}_{u}$, we allow the transit set membership to supersede the other sets, i.e., $O_{u}=\breve{O}_{u}-\breve{O}_{u} \cap \vec{T}_{u}$, etc. where $\breve{O}_{u}$ is the originally defined set without the overlapping $\vec{T}_{u}$ members removed. ${ }^{3}$ Thus the vertex sets are set partitions of $V_{u}$ where:

$$
V_{u}=O_{u} \cup I_{u} \cup L_{u} \cup Z_{u} \cup \vec{T}_{u}
$$

Also note that the sets are disjoint with empty pairwise intersections. Figure 11 shows the relative membership of vertices to these various sets as seen within our real world data set.

\subsection{Time-Constrained Path Distance Algorithm}

Using our previous time-constrained definitions of $t_{\text {first }}(x, y)$ and $t_{\text {last }}(x, y)$, we create a modified arc-based breadth first search algorithm to find a set of distances from a vertex to all other connected vertices. It is important to note that an arc-based search is much easier to implement in comparison to the more common vertex-based search. This is because, within our time-constrained search, vertices are potentially revisited due to differences in $t_{\text {first }}$ along a path, but arcs are not. Our time-constrained distance algorithm is presented as two procedures.

The procedure TimeConstrainedDistanceVeCtor is the primary procedure and returns a list of tuples containing reachable vertices and the distance to each of these vertices from the starting vertex $S r c V$ within the graph $G$. It is defined as:

procedure TimeConstrainedDistanceVector $(G, \operatorname{SrcV})$

$$
\begin{aligned}
& \text { NextPass } \leftarrow\left\{\left((\operatorname{SrcV}, Y), t_{\text {first }}(\operatorname{SrcV}, Y)\right) \forall Y(\operatorname{SrcV}, Y) \in \operatorname{Arcs}(G)\right\} \\
& \text { Distance } \leftarrow 0 \\
& \text { VisitedArcs } \leftarrow \emptyset
\end{aligned}
$$

\footnotetext{
${ }^{3}$ We believe that transit vertices are one of the most interesting vertex types, so it is important they standout when more than one definition may fit.
} 
VertexDistances $\leftarrow \emptyset$

while $\mid$ NextPass $\mid>0$ do

Distance $\leftarrow$ Distance +1

ThisPass $\leftarrow$ NextPass

NextPass $\leftarrow \emptyset$

for all $((U, V)$, Time $) \in$ ThisPass do

if $(U, V) \notin V$ isitedArcs then

VisitedArcs $\leftarrow$ VisitedArcs $\cup\{(U, V)\}$

if VertexDistances $[V]$ does not exist then

VertexDistances $[V] \leftarrow$ Distance

end if

NextPass $\leftarrow$ NextPass $\cup$ AddChildArcs $(G, V$, Time $)$

end if

end for

end while

return(VertexDistances)

end procedure

The NextPass list holds a list of arcs each with an associated $t_{\text {first }}$. The Distance variable keeps track of the current arc distance being considered as the procedure's loop iterates. This outer loop continues as long as there are arcs in the list NextPass to consider, just as a traditional breadth search algorithm would work. The loop copies NextPass into a new list ThisPass and then iterates within a nested loop over all arcs within the ThisPass list, allowing NextPass to be re-initialized for updating. Each iterated arc, if it has not been seen before, is added to the list of seen arcs, and the distance is added for the associated non-visited destination vertex. Finally, NextPass is updated with a new list of outgoing (child) arcs to explore, which are viable given the time-constraint requirements using the ADDCHILDARCS procedure below:

procedure $\operatorname{AdDChildARCs}(G, V, T$ ime $)$

NextTime $\leftarrow$ Time

NextArcSet $\leftarrow \emptyset$

ChildArcs $\leftarrow\{\forall U(V, U) \in \operatorname{Arcs}(G)\}$

for all Child $\in$ ChildArcs do

if Time $\leq t_{\text {last }}($ Child $)$ then

if NextTime $<t_{\text {first }}($ Child $)$ then

NextTime $\leftarrow t_{\text {first }}($ Child $)$

end if

NextArcSet $\leftarrow$ NextArcSet $\cup\{($ Child, NextTime $)\}$

end if

end for

return (NextArcSet)

end procedure

The execution steps with the state of the primary variables of this timeconstrained breadth first search is shown for a simple graph in Figure 12.

A traditional breadth first search for path exploration has complexity $\Theta(|V|+$ 
$|A|)$ and enables graph diameter computation in $\Theta(|V| \times(|V|+|A|))$. Our timeconstrained version, searching arcs instead of vertices, has the same complexity. However, in practice since PASes are generally sparse (as shown in Figure 7) and because many potential paths are not time compliant, we find that the timeconstrained breadth first search does better than the traditional algorithm. The differences in actual run time as compared to potential run-time complexity are shown in Table 2. One noteworthy exception is seen in the time-enforcing maximum in relation to $|V|+|A|$, which is due to a few complex PASes. The most significant PAS in question, with a $|V|+|A|$ ratio of 92.8 , had 338 vertices, 2610 arcs, multiple cycles, 5958 crossing subpaths, and required 273,620 iterations to calculate $\vec{D}_{u}$; a very rare and complex PAS.

\section{Application}

While we see a significant number of potential uses for PASes and authentication graphs generally, we next present specific examples using logistic regression over PAS attributes to show value in differentiating user types (administrators versus general users) and detecting users that have become compromised because their authentication credentials have been stolen.

Logistic regression was chosen over other potential approaches due to its model simplicity and its ability to quantify the significance of each individual attribute used within the model. Graph attributes were included within the models discussed below only if they had a $p$-value of 0.01 or less (indicating high significance within the model). Though logistic regression was a valuable method for research evaluation, more robust methods, including random forests (Breiman, 2001) or support vector machines (Hsu and Lin, 2002) may provide improved results and are an area of future work in consideration for an operational system.

\subsection{User Type Profiling}

The differences between PAS attributes are a valuable differentiator of certain user classifications within an enterprise network. More specifically, the attributes can differentiate users who have privileged administration access to computers versus those who do not. Identifying users that have administrative access can be challenging in large enterprise environments that often do not centrally manage or track such access. As was seen in the various attribute distributions discussed previously, administrative users usually have more complex PASes when compared with general users. Figure 4 shows a common administrative user within the one year data set, while Figure 2 shows a much simpler general user.

In addition to the PAS attributes defined in Section 3, we define three more attributes using histograms over various attributes of the PAS. First is an indegree histogram where each discrete value of indegree count from the PAS is a bin in the histogram. The second is based on the histogram of time-constrained path lengths. The final attribute is based on the histogram of vertex types 
within a PAS. To represent the distributional aspects of these histograms in a singular value, we use Shannon entropy defined as $\sum p \log (p)$ where $p$ is the relative frequency of each histogram bin. We find this entropy value to be a convenient single valued summary of the histogram distribution.

Our analysis shows that the following PAS attributes provide significant value in separating general users from administrative users (by having a $p$-value of 0.01 or less); this was determined by a logistic regression classifier using our previously discussed general user and administrative user labels over the one year data set:

- $\left|V_{u}\right|$ : vertex (computer) count

- $\left|A_{u}\right|$ : arc count (authentications from one computer to another)

- $\rho_{u}$ : graph density

- $\vec{D}_{u}$ : time-constrained diameter

- $\left|C_{u}\right|:$ connected component count

- $S_{u}$ : the entropy of the histogram of the PAS vertices' indegree

Let $X$ be the random variable defined by:

$$
X= \begin{cases}0 & \text { if } u \text { is not an administrator } \\ 1 & \text { if } u \text { is an administrator }\end{cases}
$$

We can define a logistic regression using the set of attributes as:

Model 1: $\log \left(\frac{P(X=1)}{P(X=0)}\right)=\beta_{0}+\beta_{1}\left|V_{u}\right|+\beta_{2}\left|A_{u}\right|+\beta_{3} \rho_{u}+\beta_{4} \vec{D}_{u}+\beta_{5}\left|C_{u}\right|+\beta_{6} S_{u}$.

For comparison purposes, we define Model 2 without time-constrained attributes, in which only vertex and arc counts are significant ( $p$-value of 0.01 or less):

$$
\text { Model 2: } \log \left(\frac{P(X=1)}{P(X=0)}\right)=\beta_{0}+\beta_{1}\left|V_{u}\right|+\beta_{2}\left|A_{u}\right| \text {. }
$$

Similarly, we define Model 3 as a simple base model with only PAS connected component count:

$$
\text { Model 3: } \log \left(\frac{P(X=1)}{P(X=0)}\right)=\beta_{0}+\beta_{1}\left|C_{u}\right| \text {. }
$$

The resulting logistic regression classifiers show good results in differentiating the two user types. We find that the attributes that were most significant in the models match our assumptions. As expected, graph size and complexity as represented by vertex and arc counts, density, and diameter are strongly represented within the model results.

As seen in Figure 13, we can predict a system administrator approximately $80 \%$ of time with approximately a 1 in 8 false positive rate with the Model 1 
feature set. The Receiver Operating Characteristic (ROC) curve (Bradley, 1997) also shows the two other models result in less accuracy. To assist in validating our models, we used 10-fold cross validation to generate the average ROC curves. More specifically, we randomly divided the data into ten equal subsets where one subset was used as validation against the other nine. The resulting ROC curve is the average across the non-validating nine subsets of the data in comparison to the tenth validation subset. Additional details of $k$-fold validation can be found in Kohavi (1995).

Table 3 shows the details of the logistic regression classifiers for all three models including coefficients and their significance. Note again that every coefficient used is statistically significant at the 0.01 level or better, thus reduced models such as Model 2 and Model 3 do not fit as well. Yet we present Model 2 and Model 3 as simpler models for both comparison and possible circumstances where fewer graph attributes are available due to constrained computation or other limitations.

Of particular interest are the few outlying users with large and complex graphs who are not administrators and are responsible for most of the false positives within our model. These users may simply be defined as "power users" or administrators that were missed in our classification method. While unlikely, inappropriate behavior may also be exhibited by some of these users (or by someone else misusing their tickets/credentials). These users require further investigation and research. One of the advantages to the use of PAS attributes is that it can help focus limited analyst time on users whose activity requires further investigation.

The analysis of why administrators have more complex and extensive graphs is ongoing, but some likely reasons can be hypothesized. For example, because administrators often manage a large number of computers, they are likely to $\log$ into many or all of those computers as a function of their job. They also rely on various network computers, like central patch servers or application install servers, that increase their authentication graph diameter and maximum indegree.

We believe that other user differentiation may be possible through PAS characteristics. For example, PAS attributes were used to differentiate general users, administrators, and a subset of "core" institutional administrators by (Kent and Liebrock, 2013), though without the use of time-constrained attributes. In comparison, the computationally less expensive time-constrained attributes used in this paper generated comparable or slightly better differentiation results. In addition, the data set used in the previous work was from a different, non-overlapping time period.

\subsection{Detection of Compromise}

PASes may be used to detect network compromises that involve inappropriate use of a user's authentication credentials. There is a set of graph attributes that differentiate what most normal users do as a function of their authentication activity from what a malicious entity would do with the same credentials, at least in our studied data set. 
As a basis for validation, we have a complete set of data surrounding a red team exercise that spanned a 1 month time period, as presented in Section 2. The exercise simulated a malicious attack where a set of users were spear phished via email ${ }^{4}$ and a small number had their user accounts compromised including their associated authentication credentials. The red team then was able to expand their presence within the network through lateral credential exploitation and credential theft (Hummel, 2009), gaining access to 81 different users' authentication credentials across many more computers within the network. We have labeled these 81 users as compromised for the purposes of differentiating them from our much larger population of non-compromised users. It is worth noting that 40 of these compromised users were also labeled as administrators, as discussed in Section 4.1, showing the value of targetting of administrator-level credentials by the red team.

Similar to our approach with administrative users and general users, we used a logistic regression classifier to determine that the following set of PAS attributes provide value in separating compromised users from non-compromised users (by having a $p$-value of 0.01 or less):

- $\left|V_{u}\right|$ : vertex (computer) count

- $\left|A_{u}\right|:$ arc count (authentications from one computer to another)

- $\rho_{u}$ : PAS density

- $\vec{D}_{u}$ : time-constrained diameter

- $S_{u \vec{P}}$ : the entropy of the histogram of the PAS time-constrained path lengths

- $S_{u V T}$ : the entropy of the histogram of vertex types $\left(I_{u}, O_{u}, L_{u}, Z_{u}, \vec{T}_{u}\right)$ within the PAS

Let $X$ be the random variable such that:

$$
X= \begin{cases}0 & \text { if } u \text { is not compromised } \\ 1 & \text { if } u \text { is compromised }\end{cases}
$$

The logistic regression for the full model, Model 1, using all of the relevant, statistically significant attributes is:

$$
\text { Model 1: } \begin{aligned}
\log \left(\frac{P(X=1)}{P(X=0)}\right)= & \beta_{0}+\beta_{1}\left|V_{u}\right|+\beta_{2}\left|A_{u}\right|+\beta_{3} \rho_{u}+\beta_{4} \vec{D}_{u} \\
& +\beta_{5} S_{u \vec{P}}+\beta_{6} S_{u V T}
\end{aligned}
$$

\footnotetext{
${ }^{4}$ Spear phishing is a malicious social engineering exploit where a user is convinced by the contents of an email to take some action that allows the malicious actor to take control of that user's computer environment and associated account.
} 
For comparison purposes, we define Model 2 using non-time-constrained, yet still statistically significant attributes:

$$
\text { Model 2: } \begin{aligned}
\log \left(\frac{P(X=1)}{P(X=0)}\right)= & \beta_{0}+\beta_{1} \rho_{u}+\beta_{2} D_{u} \\
& +\beta_{3} S_{u P} .
\end{aligned}
$$

Similarly, we define Model 3 using only non-time-constrained diameter:

$$
\text { Model 3: } \log \left(\frac{P(X=1)}{P(X=0)}\right)=\beta_{0}+\beta_{1} D_{u} .
$$

Finally, we define Model 4 with just the graph density as a base comparison:

$$
\text { Model 4: } \log \left(\frac{P(X=1)}{P(X=0)}\right)=\beta_{0}+\beta_{1} \rho_{u} \text {. }
$$

The resulting classifiers show a useful ability to detect the difference between compromised and non-compromised users. Similar to user type differentiation, we find that the attributes that were most significant in the models match our assumptions. As expected, graph size and complexity as reflected in the graph attributes are different between compromised and non-compromised users. For example, we know that diameter often increases when users become compromised.

As seen in Figure 14, we can use logistic regression Model 1 to successfully detect a compromise approximately $28 \%$ of the time with only a 1 in 800 false positive rate. This particularly low false positive rate is very important given a user population size of approximately 12,000 where security analysts are required to examine all compromise indicators, true or false. The figure also shows three other models that result in less accuracy and include the use of non-timeconstrained attributes. Again, 10-fold cross validation was used to generate the average ROC curves. Table 4 shows the logistic regression classifier fits for all 4 models including coefficients and their variable significance.

Although a $28 \%$ detection rate seems low in comparison to many existing techniques for detecting traditional compromise events, we believe the ability to detect actual credential theft and resultant credential hopping is novel and difficult to compare with other intrusion detection techniques that look for more well-defined compromise indicators (discussed in Section 6). Since most relevant, real-world attacks compromise multiple credentials, detecting any one stolen credential is sufficient to detect the overall attack. Thus, if we assume that individual detections are statistically independent and have the same probability $p$ of detection, then the probability of detecting the overall attack is $1-\left((1-p)^{n}\right)$, where $n$ is the number of individual detections. In the red team example, with $p=0.28$ and $n=81$, the overall detection probability is $100 \%$ to eleven decimal places. In a more realistic phishing email attack with lateral network movement using stolen credentials, where the attacker compromises only 8 different user credentials, we would have a $93 \%$ detection rate for the overall attack. 
The logistic regression approach shows that there are distinguishing attribute combinations that differentiate compromised PASes from non-compromised. It also offers the advantages of both computational simplicity in detection and interpretability of the parameter fits. We see future opportunities to refine a classifier through the use of more descriptive graph attributes that are yet to be determined, as well as other classification approaches beyond logistic regression. Another key need for a realistic detector is to consider the data as a continuous time series of activity.

\subsection{Detection Through Time Series Analysis}

Most cyber security data sets, including the authentication data sets discussed throughout this paper, are sequences of time series events. Representing these events as graphs provides relational insights and feature extraction opportunities, but also lead to a loss of dynamic time characteristics that may be present in the data. As a result, there is a need to consider PASes in terms of a time series of dynamic activity. The time-constrained features discussed in Section 3 are one aspect of meeting this need but do not fully account for continuous time dynamics.

Our primary approach with dynamic time series graph analysis has been to create PASes over sliding time windows of authentication events. We have found 2 hour time windows, sliding by 30 minutes, provide a minimum window length; shorter time periods result in PASes that are too sparse. Further investigations of relevant time periods is a subject for future work.

Our exploration into comparing PASes in these moving time windows has yielded interesting preliminary results. For example, we have found that our administrative users have bimodal behavior. In some time periods, their PASes reflect that of a general user and in other time periods we see that they have the more complicated graph we expect to see from administrative users. We believe that this can be explained by the fact that administrative users sometimes have the behavior of general users. This differentiation can been seen in Figure 15, where there is an overall increased separation between the standard deviation of arc counts in administrative users in comparison to general users. This difference represents the variability increase observed in administrative users. We find that compromised users have even more variability.

Time series analysis over PASes also makes sense for intrusion detection. To this end, we find that there are key features that effectively detect compromise events within time series PASes; at least in some cases. Figure 16 shows a time series of $\vec{D}_{u}$ for two different users. While the ability to detect the compromise activity is present using just the diameter attribute, there are significant false positives with this simplified approach. Using more integrated feature sets across the time series generated PASes, in combination with more nuanced comparison techniques, will enable better approaches for determining inappropriate use of authentication credentials. Per user models, normalized to their past behavior, should yield improved detection quality. For example, an exponentially weighted moving average on the diameter, combined with a secondary cumulative sum or 
CUSUM (Barnard, 1959) of the diameters' $p$-values, would detect both shortterm and longer-term anomaly events over time. The CUSUM is a method borrowed from the control chart literature for handling both acute, single point anomalies, along with moderate anomalies that occur over the recent past. It is a lightweight, easily update method for accounting for dependent anomalous data over time. We believe this approach would provide a computationally feasible, real-time detection capability based on the work from Lambert and Liu (2006).

We also see an opportunity to explore continuously updating PASes that add and remove vertices and arcs and enable real-time analysis through a dynamic process. This would improve upon our current time window approach and potentially increase the fidelity and usefulness of time-constrained aspects of PASes.

\section{Bipartite Authentication Graphs}

In addition to PASes, we can also use authentication data to generate a bipartite graph where the graph vertices are the two disjoint sets of users and computers. This bipartite graph then associates the user and computer sets with edges representing authentication events. More specifically, we can define a bipartite authentication graph (BAG) for all user authentication activity in an enterprise network as $B=(U, V, E)$ where $U$ is a set of vertices that represent users authenticating in the network, $V$ is a set of vertices that represent computers that users are authenticating to, and $E$ represents a set of undirected edges that associates users from $U$ authenticating to (or on) a computer in $V$ over some time period. This creates an affiliation network (Breiger, 1974) between users and computers on the basis of authentication activity. This representation allows us to explore the relationship and significance of credentials and their use over communities of computers and users. This representation may also open a variety of research opportunities relating to social network affiliation analysis and how people use computer networks.

For our current work, we are using the bipartite graph representation as an effective method to examine potential risk of credential stealing and compromise within the enterprise network. Since this bipartite graph captures the entire user population and their relationships through computers, there is the potential to identify credential theft and hopping, which is unavailable in the previously discussed PAS analysis. A key question we can ask is what user or computer represents the most risk of compromise within the network. In simple form we can ask this by considering the vertex with the highest degree. A more exact approach would be to find the vertex that, if removed, increases the connected component count the most for the overall BAG. In most cases, we found that these two measures result in the same vertex selection. An increase in the connected component count potentially makes it more difficult for a malicious actor to steal and reuse credentials to move through a network. An increase in connected component count within a network can be equated to a reduction 
in the risk associated with credential theft and subsequent credential hopping within the network.

For our BAG analysis, we have found that shorter time periods of 1 day and less are the most useful due to better matching to credential life times (Kerberos tickets generally have life spans of 1 day or less). For example, when we build a BAG over a typical 1 day time period from our larger data set, we generate a graph with 8699 users and 10,141 computers. It has a connected component count of 1426 with the majority of users and computers being in the primary component. When we remove the user vertex that has the highest degree (132), we increase the component count by 38. A simplified visualization of this change is shown in Figure 17. Interestingly, when we consider the BAGs from time periods prior to the month where we have red team compromise data, we find that the top two connecting users by degree were both compromised. One of the users was a system (non-person) account used to do health monitoring over a large set of computers. As a result of our identification through BAG analysis, this system account has been operationally broken up into multiple system accounts that reduce the risk and impact of compromise.

\section{Related Work}

The IDES work by Denning (1987) and NIDES work by Anderson et al. (1995) are early examples of intrusion detection systems that used host event data to classify normal and intrusion-related behaviors. The data sources used, while more diverse then just authentication data, did not consider the concept of movement or traversal between computers. In general, we find that most existing approaches in the literature either consider intrusion detection techniques specific to individual host or consider very generic network data for network intrusion detection.

One area of relevant work can be found in the masquerade detection literature, which involves the detection of user behavior outside of the user's normal envelope. Salem et al. (2008) provide a good summary of existing masquerade detection methods up to 2008. In much of that work, host-based views of the data take center stage. Sequences of Unix or Windows shell commands, file accesses, system calls, registry accesses, etc. are examined, and various machine learning techniques are applied. Since these approaches analyze hosts independently, and our work focuses on analysis of the behavior of multiple hosts acting together, the two are not easily compared.

The system developed by Maloof and Stephens (2007), called ELICIT, is based upon the idea that even if users have authorization to access documents, files, servers, etc., they may not have need to know, and therefore, by using a social network approach, they define need-to-know for individual users, and identify violations therein. While this does indeed capture graph properties, the development of need-to-know per user is involved, requiring the merging of several data sets, any of which may be difficult to obtain in real time. Our work, conversely, uses logs readily available from the Active Directories, making it more attractive from a deployment standpoint. 
Since 2008, more work has been done on masquerade detection, generally focused on host-based data (Guan et al., 2009) or single user behavior (Salem and Stolfo, 2009). Network-observable masquerade literature is more scarce. In Menon and Pottenger (2009), the authors examine BGP data sets (not internal network data), and network file system calls to establish user profiles. The latter is more relevant to the current work, but still does not examine multiple users at once to discover if there is a masquerader moving amongst them.

Generally, existing intrusion detection systems do not consider the use of stolen network authentication credentials, which from the view of most traditional detection approaches, looks like normal computer and network activity. One exception is the Heat-ray system described by Dunagan et al. (2009), where predefined attack graphs combined with machine learning are used to build security configurations within a network of computers that reduce the potential for network authentication credential hopping (described as snowballing within the paper) due to credential theft. This work does not address the detection of credential hopping. While reducing the potential for credential hopping is extremely important, we believe that credential hopping will continue to be a significant issue in large-scale network authentication environments, even with configuration improvements.

Using graphs to help detect inappropriate insider activity from various data sets beyond just computer network activity has been explored (Eberle et al., 2010). This work, while relevant, assumes an isomorphic subgraph construct for defining anomalous behavior that is both difficult to use in our observed noisy cyber network environments and potentially computationally expensive across many user graphs over many time frames. The work by Chen et al. (2012) uses graph analysis from collaborative information system data sources to detect potentially anomalous insider activity.

We believe that our research is the first to represent and analyze network user authentication activity as a graph and a set of dynamic graph attributes. However, there is a foundation of related work using graph analysis for intrusion detection, insider threat analysis, and network behavior analysis.

The GrIDS system developed by Staniford-Chen et al. (1996) is an early example of using graphs for the purposes of large network cyber intrusion detection. They generated activity graphs representing network communication enabling extensible detection of anomalous edge or vertex sets and policy enforcement as a function of a graph structure. In addition, this is an early example where it is shown that graphs provide a valuable visual representation of potentially harmful activity. The work by Ammann et al. (2002) demonstrates the use of simple but large graphs in network vulnerability analysis. Perhaps somewhat similar to our research, the work by Hogan et al. (2013) shows a method of graph abstraction using graph minors over a path to help predict and detect lateral movement within large networks. Unfortunately, this work did not contain results that enable comparison. The work by Djidjev et al. (2011) introduces the concept of temporal constraints on paths to help discover anomalous movement within a network.

Attack graphs are another area of related anomaly detection worth noting. 
An attack graph is a static representation of all paths through a network that end in nodes where an intruder has successfully achieved his goal (Jha et al., 2002). Zhang et al. (2008) proposed an one-step worst attack graph method. With the attack graph generated by his algorithm, network alerts can be correlated into scenarios jointly based on graph distance and time gap between candidate alerts. Wang and Daniels (2008) demonstrates evidence graphs for automated evidence analysis. The evidence graphs are generated with each node representing a host of interest and each edge an observed intrusion. Based on these evidence graphs, the author proposed a reasoning framework which decides the state of a host (attacker, victim, transitive/stepping-stone or affiliated) and the relationship between several hosts to discover the attack source. Similarly using existing attack data, Ha et al. (2007) show a method for analyzing network attacks using Capability Acquisition Graphs (CAGs), which help to model and deconstruct the complexity involved in insider attack analysis. Unfortunately, these attack graph and evidence-based approaches assume that the attack structure can be pre-determined, an assumption that may be appropriate in a post-detection, forensic situation, but not immediately relevant or comparable to our work.

A variety of work involving relevant graph analysis has also been done on the Enron email data corpus (Shetty and Adibi, 2005; Chapanond et al., 2005). However, a particularly relevant research study by Priebe et al. (2005) involves the use of scan statistics to analyze a set of time series graphs representing Enron email. This work introduces the importance of subgraph characteristics as features to consider over the time series. Similarly, the work by Neil et al. (2013) extends and generalizes the scan statistic concept over a time series of paths within a network to detect inappropriate network movement. Subgraph pattern analysis over a time series is discussed by Borgwardt et al. (2006). The work by Macindoe and Richards (2010) provides a very interesting and relevant method of comparing graph similarity based on various graph feature distributions that encompasses internal subgraph structure.

\section{Future Work}

Looking forward, we see a continued opportunity for using user authentication graphs and bipartite graphs for analysis, user profiling, and visual representation. First, we see the need to move to time series analysis on the authentication graphs. While our static analysis has proven valuable, we see increased opportunity, fidelity, and practicality with various time series approaches as discussed in Section 4.3. One particularly interesting analysis would be to measure individual user graph variability over time, allowing us to use authentication graphs to detect malicious insiders as well as compromised user accounts. Next, we believe there would be significant value in understanding what outlier users are actually doing, as previously discussed. Understanding additional user type categories or subcategories beyond the two considered in this paper may be very useful. Finally, we see opportunities to mix the authentication graph characteristics with other user network behavior measures. For example, work has 
begun to integrate web browsing behaviors (Kent et al., 2013) with the user authentication graph attributes to potentially predict and prevent computer and network compromise events that are visible in the combined data set. Because computing the graph attributes for PASes and the associated models is relatively straight-forward, we believe continuous, real-time detection and analysis is a viable future capability, which we are pursuing.

We see continued authentication graph, PAS, and BAG feature exploration and feature selection as future areas of focus. In addition, more fine-grained approaches to graph comparison need to be considered for user differentiation. Improved graph comparison methods will also support better detection of credential compromise and other inappropriate user activity, including insider threat.

Another key future need is to validate our approaches and results on additional data sources and attack events beyond LANL's network. While we believe the LANL network is generally representative of most enterprise-scale networks that use centralized authentication, such a statement is speculative. Though rare, additional data sets associated with compromised authentication credentials would also be useful in validating and extending the results we have presented. We hope that the results and opportunity we have shown will motivate other organizations to collect the data and apply the techniques we have discussed, enabling both cross-validation and opportunities for continued research.

\section{Conclusion}

This paper has demonstrated a useful and interesting way to examine and analyze large-scale user authentication activity within a centralized network authentication system. It has shown initial value in differentiating administrative and general users as well as finding compromised users within an enterprise network. In addition, we demonstrated a method of objectively representing how authentication activity across users and computers can increase the risk of credential loss and how mitigation scenarios effect such risk.

We see the concepts and work presented in this paper as a foundation for significant and fruitful future research relevant to cyber defense needs and perhaps many other operational IT requirements.

\section{Acknowledgments}

A number of people contributed to the success of this research. First, we would like to thank Aric Hagberg for pointing out the simplicity of arc traversal over vertex traversal for our time-constrained breadth-first search algorithm. We appreciate Subhashish Mazumdar's help in reviewing and validating the timeconstrained algorithm. Finally, we are grateful for the reviews and suggestions from Aric, Pieter Swart, Nathan Lemons, Mike Fisk, and Feng Pan. This work was funded, in part, by the Department of Energy. 


\section{References}

Ammann, P., Wijesekera, D., and Kaushik, S. (2002). Scalable, graph-based network vulnerability analysis. In Proceedings of the 9th ACM Conference on Computer and Communications Security, pages 217-224. ACM.

Anderson, D., Frivold, T., and Valdes, A. (1995). Next-generation intrusion detection expert system (NIDES): A summary. SRI International, Computer Science Laboratory.

Bang-Jensen, J. and Gutin, G. Z. (2009). Digraphs: theory, algorithms and applications. Springer.

Barnard, G. A. (1959). Control charts and stochastic processes. Journal of the Royal Statistical Society. Series B (Methodological), pages 239-271.

Borgwardt, K., Kriegel, H.-P., and Wackersreuther, P. (2006). Pattern mining in frequent dynamic subgraphs. In Proceedings of the Sixth International Conference on Data Mining, pages 818-822.

Bradley, A. P. (1997). The use of the area under the ROC curve in the evaluation of machine learning algorithms. Pattern recognition, 30(7):1145-1159.

Breiger, R. L. (1974). The duality of persons and groups. Social Forces, 53(2):181-190.

Breiman, L. (2001). Random forests. Machine learning, 45(1):5-32.

Chapanond, A., Krishnamoorthy, M., and Yener, B. (2005). Graph theoretic and spectral analysis of Enron email data. Computational and Mathematical Organization Theory, 11(3):265-281.

Chen, Y., Nyemba, S., Zhang, W., and Malin, B. (2012). Specializing network analysis to detect anomalous insider actions. Security Informatics, 1(1):1-24.

Denning, D. (1987). An intrusion-detection model. Software Engineering, IEEE Transactions on, SE-13(2):222-232.

Djidjev, H., Sandine, G., Storlie, C., and Vander Wiel, S. (2011). Graph based statistical analysis of network traffic. In Proceedings of the Ninth Workshop on Mining and Learning with Graphs.

Dunagan, J., Zheng, A. X., and Simon, D. R. (2009). Heat-ray: Combating identity snowball attacks using machine learning, combinatorial optimization and attack graphs. In Proceedings of the Symposium on Operating Systems Principles, pages 305-320. ACM.

Eberle, W., Graves, J., and Holder, L. (2010). Insider threat detection using a graph-based approach. Journal of Applied Security Research, 6(1):32-81. 
Fisk, M. E. (2014). Filemap:map-reduced program execution on loosely-coupled distributed systems. In Proceedings of the Fourth International Workshop on Cloud Data and Platforms.

Glass, E. (2006). The NTLM authentication protocol and security support provider. http://davenport. sourceforge. net/ntlm.html.

Guan, X., Wang, W., and Zhang, X. (2009). Fast intrusion detection based on a non-negative matrix factorization model. Journal of Network and Computer Applications, 32(1):31-44.

Ha, D., Upadhyaya, S., Ngo, H., Pramanik, S., Chinchani, R., and Mathew, S. (2007). Insider threat analysis using information-centric modeling. Advances in Digital Forensics III, pages 55-73.

Hogan, E., Johnson, J. R., and Halappanavar, M. (2013). Graph coarsening for path finding in cybersecurity graphs. In Proceedings of the Eighth Annual Cyber Security and Information Intelligence Research Workshop, CSIIRW '13, pages 7:1-7:4, New York, NY, USA. ACM.

Hsu, C.-W. and Lin, C.-J. (2002). A comparison of methods for multiclass support vector machines. Neural Networks, IEEE Transactions on, 13(2):415425 .

Hummel, C. (2009). Why crack when you can pass the hash? SANS Institute InfoSec Reading Room.

Jha, S., Sheyner, O., and Wing, J. (2002). Two formal analyses of attack graphs. Proceedings 15th IEEE Computer Security Foundations Workshop, pages $49-63$.

Johnson, A., Dempsey, K., Ross, R., Gupta, S., and Bailey, D. (2010). NIST guide for security configuration management of information systems. http://csrc.nist.gov/publications/drafts/800-128/draft_ sp800-128-ipd.pdf.

Kent, A. and Clifford, J. (2000). Network information management and distribution in a heterogeneous and decentralized enterprise environment. In Proceedings of the 14th USENIX Conference on System Administration, pages 85-94. USENIX Association.

Kent, A. D. and Liebrock, L. M. (2013). Differentiating user authentication graphs. In 2nd Annual IEEE CS Security and Privacy Workshop on Research of Insider Threat WRIT.

Kent, A. D., Liebrock, L. M., and Neil, J. (2013). Web adoption: An attempt toward classifying risky internet web browsing behavior. In Proceedings of the Learning from Authoritative Security Experiment Results (LASER) Conference. 
Kohavi, R. (1995). A study of cross-validation and bootstrap for accuracy estimation and model selection. In Proceedings of the International Joint Conference on Artificial Intelligence, volume 14, pages 1137-1145.

Kumar, V. (1992). Algorithms for constraint-satisfaction problems: A survey. AI Magazine, 13(1):32.

Lambert, D. and Liu, C. (2006). Adaptive thresholds: monitoring streams of network counts. Journal of the American Statistical Association, 101(473):7888.

Macindoe, O. and Richards, W. (2010). Graph comparison using fine structure analysis. In Proceedings of the IEEE Second International Conference on Social Computing, pages 193-200.

Maloof, M. A. and Stephens, G. D. (2007). elicit: A system for detecting insiders who violate need-to-know. In Recent Advances in Intrusion Detection, pages 146-166. Springer.

Menon, V. and Pottenger, W. M. (2009). A higher order collective classifier for detecting and classifying network events. In Intelligence and Security Informatics, 2009. ISI'09. IEEE International Conference on, pages 125-130. IEEE.

Neil, J., Storlie, C. B., Hash, C., Brugh, A., and Fisk, M. (2013). Scan statistics for the online detection of locally anomalous subgraphs. Technometrics, Accepted, in prepublication.

Neuman, B. and Ts'o, T. (1994). Kerberos: An authentication service for computer networks. Communications Magazine, IEEE, 32(9):33-38.

Priebe, C. E., Conroy, J. M., Marchette, D. J., and Park, Y. (2005). Scan statistics on enron graphs. Computational and Mathematical Organization Theory, 11(3):229-247.

Salem, M. B., Hershkop, S., and Stolfo, S. J. (2008). A survey of insider attack detection research. Insider Attack and Cyber Security, pages 69-90.

Salem, M. B. and Stolfo, S. (2009). Masquerade attack detection using a searchbehavior modeling approach.

Shetty, J. and Adibi, J. (2005). Discovering important nodes through graph entropy the case of Enron email database. In Proceedings of the 3rd International Workshop on Link Discovery, LinkKDD '05, pages 74-81, New York, NY, USA. ACM.

Staniford-Chen, S., Cheung, S., Crawford, R., Dilger, M., Frank, J., Hoagland, J., Levitt, K., Wee, C., Yip, R., and Zerkle, D. (1996). GrIDS-a graph based intrusion detection system for large networks. In Proceedings of the 19th National Information Systems Security Conference, volume 1, pages 361-370. Baltimore. 
Wang, W. and Daniels, T. (2008). A graph based approach toward network forensics analysis. ACM Transactions on Information and System Security, 12(1):1-33.

Zhang, S., Li, J., Chen, X., and Fan, L. (2008). Building network attack graph for alert causal correlation. Computers \& Security, 27(5-6):188-196. 


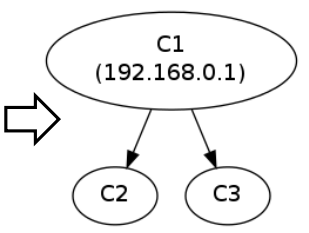

Figure 1: Two example logged events for user U1 where computer access was granted, one for computer IP address 192.168.0.1 (or computer C1) access to computer C2 and another for the same IP address access to computer C3. The induced directional graph from these two log messages is also shown. If this accounts for all of the authentication activity in a given time period for user U1, it would be the user's authentication subgraph as discussed in Section 3.

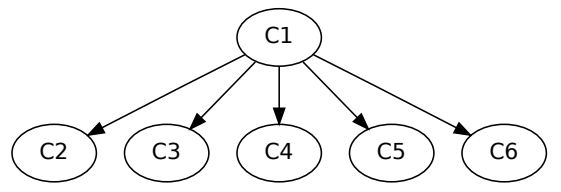

Figure 2: The network authentication graph for a typical general user $u$ in the 1 year authentication data set. This user accessed 6 computers $\left(\left|V_{u}\right|=6\right)$ with a main computer (normally the user's desktop) connecting to 5 other computers $\left(\left|A_{u}\right|=5\right)$. Note that this creates a graph of diameter $1\left(D_{u}=1\right)$, a maximum outdegree of $5\left(\max \left(\operatorname{deg}^{+}\right)=5\right)$, and a maximum indegree of $1\left(\max \left(\mathrm{deg}^{-}\right)=1\right)$. Graph notion is defined and described in Section 3.

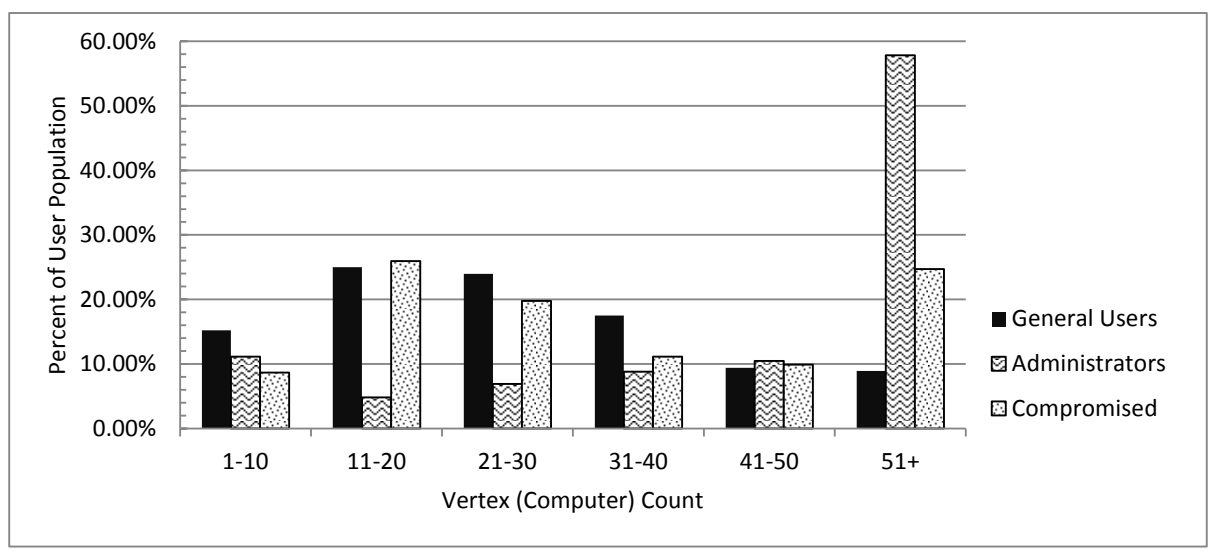

Figure 3: Histogram of vertex counts $\left(\left|V_{u}\right|\right)$ across general users, administrative users, and users that were compromised for the full one year period. 
Figure 4: The network authentication graph $G_{u}$ from a typical user $u$ with administrative access. This user accessed 18 computers $\left(\left|V_{u}\right|=18\right)$ with 41 authentication $\operatorname{arcs}\left(\left|A_{u}\right|=41\right)$. Note that this graph has a diameter of $5\left(D_{u}=5\right)$ and a time-constrained diameter of 4 $\left(\vec{D}_{u}=4\right)$. It represents a more complex authentication graph when compared with most general users.

\begin{tabular}{|l|l|}
\hline Variable & Description \\
\hline$G_{u}$ & Person's Authentication Subgraph (PAS) for user $u$ \\
$v$ & Graph vertex \\
$V_{u}$ & Vertex set for PAS $u$ \\
$\left|V_{u}\right|$ & Number of vertices in PAS $u$ \\
$(x, y)$ & Graph arc connecting vertex $x$ to vertex $y$ \\
$A_{u}$ & Arc set for PAS $u$ \\
$\left|A_{u}\right|$ & Number of arcs in PAS $u$ \\
$P_{u}\left(v_{1}, v_{n}\right)$ & An arc path from vertex $v_{1}$ to vertex $v_{n}$ in PAS $u$ \\
$\left|P_{u}(x, y)\right|$ & A path length from $x$ to $y$ in PAS $u$ \\
$d(x, y)$ & Shortest path distance from $x$ to $y$ \\
$D_{u}$ & Graph diameter of PAS $u$ \\
$\vec{P}_{u}\left(v_{1}, v_{n}\right)$ & Time-constrained arc path in PAS $u$ \\
$\vec{d}_{(}(x, y)$ & Time-constrained path distance \\
$\vec{D}_{u}$ & Time-constrained graph diameter of PAS $u$ \\
$\rho_{u}$ & Directed graph density of PAS $u$ \\
$C_{u}$ & Connected component set of PAS $u$ \\
$\left|C_{u}\right|$ & Connect component count of PAS $u$ \\
$d e g$ & Indegree of vertex $v$ \\
$d e g^{+}(v)$ & Outdegree of vertex $v$ \\
$O_{u}$ & Outstar vertex set for $u$ \\
$I_{u}$ & Instar vertex set for PAS $u$ \\
$Z_{u}$ & Isolated vertex set for PAS $u$ \\
$\vec{T}_{u}$ & Transit vertex set for PAS $u$ \\
$L_{u}$ & Pseudo-leafe vertex set for PAS $u$ \\
$S_{u}$ & Entropy of indegree values for PAS $u$ \\
$S_{u} \vec{P}$ & Entropy of time-constrained path lengths for PAS $u$ \\
$S_{u V T}$ & Entropy of vertex type counts for PAS $u$ \\
\hline
\end{tabular}

Table 1: List of variables and associated descriptions. 
Figure 5: An example graph where $P\left(v_{1}, v_{4}\right)$ is the longest non-time-constrained path, with a length of 3 , and $\vec{P}\left(v_{1}, v_{3}\right)$ is the longest valid time-constrained path, with a length of 2 . More specifically, $\vec{P}\left(v_{1}, v_{4}\right)=\emptyset$ because $t_{\text {last }}\left(v_{3}, v_{4}\right)<t_{\text {first }}\left(v_{2}, v_{3}\right)$.

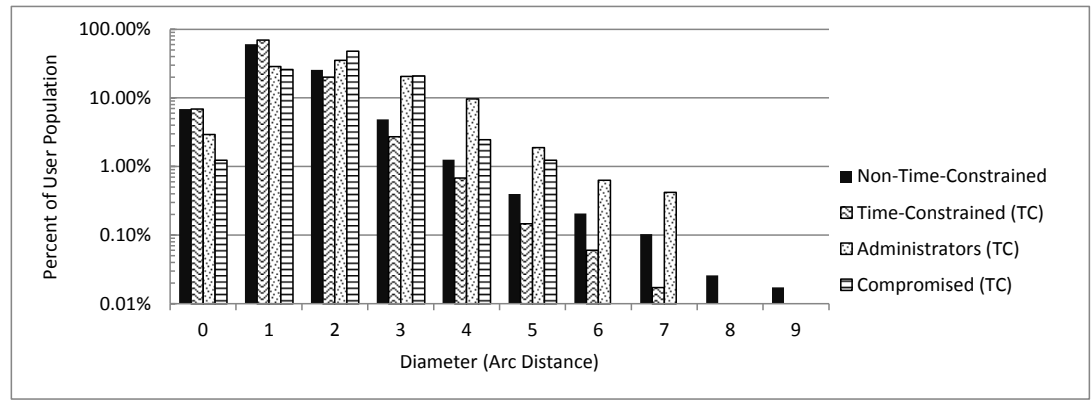

Figure 6: Histogram of both traditional graph diameter $D_{u}$ (non-time-constrained) and timeconstrained diameter $\vec{D}_{u}$ (TC) for general users, administrative users, and users that were compromised. As shown, the time-constrained diameter reduces the observed diameter, particularly at the higher values.

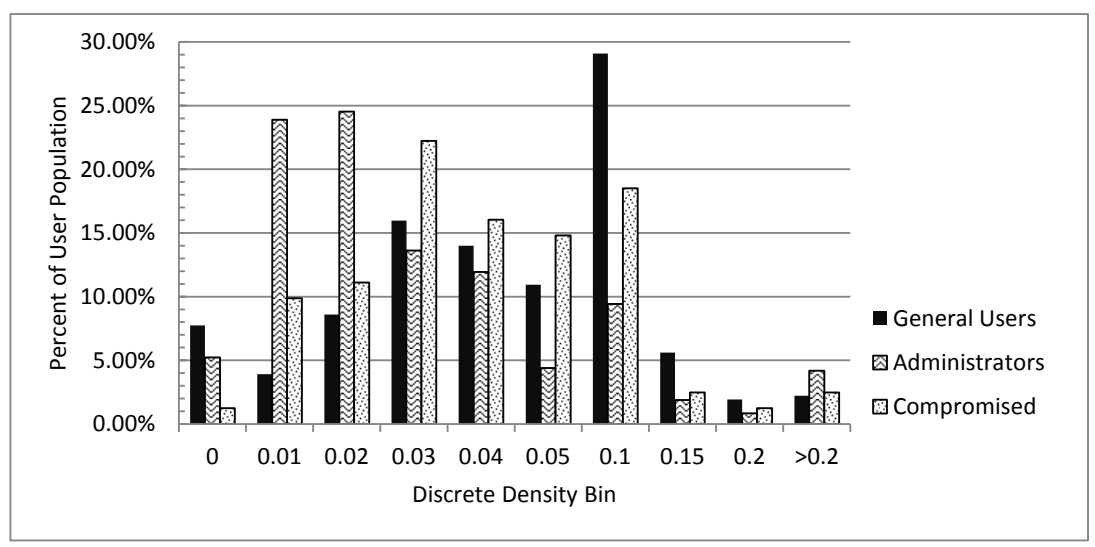

Figure 7: Histogram of discretized directed graph density, $\rho_{u}$ across general users, administrative users, and users that were compromised. 
Figure 8: Histogram of the connected component count $\left(\left|C_{u}\right|\right)$ of general users, administrative users, and users that were compromised.

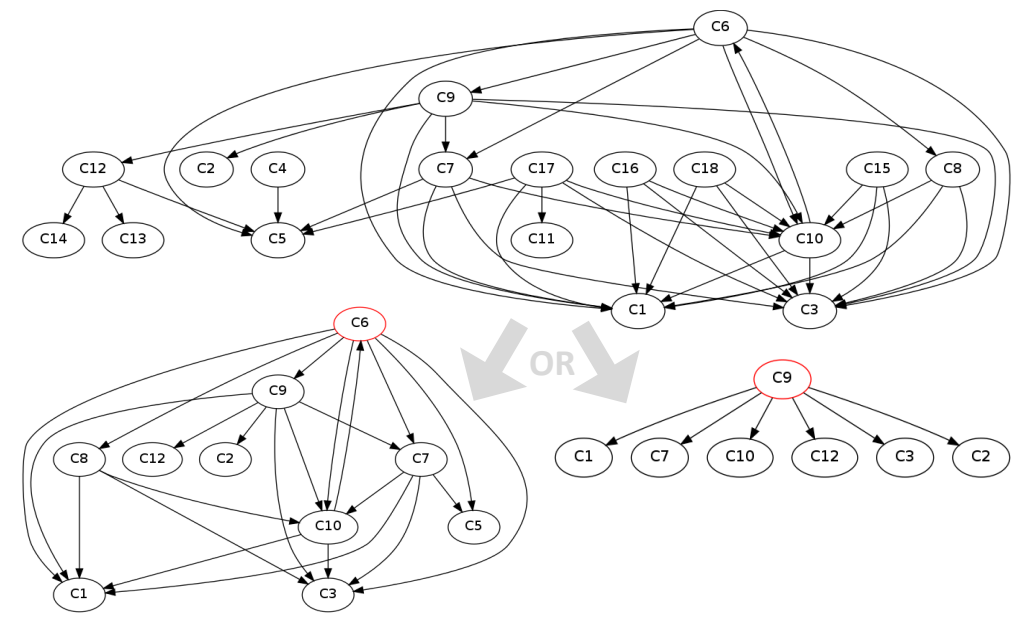

Figure 9: The PAS $G_{u}$ from of a typical user with administrative access showing how time constraints on paths $\left(\vec{P}_{u}\right)$ provide visualization simplification. This PAS is the same as shown in Figure 4. However, the bottom two graphs show two simplified views using time-constrained paths originating from two different computers (vertices) C6 and C9.

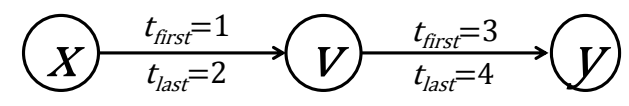

Figure 10: An example graph with a transit vertex $v$ given $t_{\text {first }}(x, v)<t_{\text {last }}(v, y)$. 


\begin{tabular}{|c|c|c|c|}
\hline Iteration & NextPass & VisitedArcs & VertexDistances \\
\hline 0) & $\begin{array}{l}\{((\mathrm{A}, \mathrm{B}), 5), \\
((\mathrm{A}, \mathrm{C}), 1)\}\end{array}$ & $\varnothing$ & $\varnothing$ \\
\hline 1) & $\{((\mathrm{C}, \mathrm{B}), 2)\}$ & $\begin{array}{l}\{(A, B), \\
(A, C)\}\end{array}$ & $\begin{array}{l}\{\mathrm{B}: 1, \\
\mathrm{C}: 1\}\end{array}$ \\
\hline 2) & $\{((B, D), 3)\}$ & $\begin{array}{l}\{(A, B), \\
(A, C), \\
(C, B)\}\end{array}$ & $\begin{array}{l}\{\mathrm{B}: 1, \\
\mathrm{C}: 1\}\end{array}$ \\
\hline 3) & $\varnothing$ & $\begin{array}{l}\{(A, B), \\
(A, C), \\
(C, B), \\
(B, D)\}\end{array}$ & $\begin{array}{l}\{\mathrm{B}: 1, \\
\mathrm{C}: 1, \\
\mathrm{D}: 3\}\end{array}$ \\
\hline
\end{tabular}

Figure 11: Histogram for all of the defined vertex types across general user, administrative users, and users that were compromised.

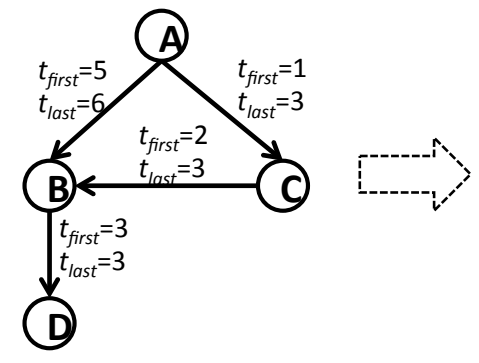

Figure 12: An example graph and the resulting steps and variables as the time-constrained distance algorithm TimeConstrainedDistanceVector is on run on the graph starting at vertex A. Note that there is one non-viable and one viable path from vertex A to vertex $D$ and that vertex $B$ is part of the exploration twice within the breadth first search. 


\begin{tabular}{|l||l|l|l|}
\hline Ratio & Traditional & $\begin{array}{l}\text { Non-time-constrained } \\
\text { Diameter } D\end{array}$ & $\begin{array}{l}\text { Time-constrained } \\
\text { Diameter } \vec{D}\end{array}$ \\
\hline$D$ Maximum & 41.4 & 3.0 & 1.0 \\
$\vec{D}$ Maximum & 92.8 & 0.67 & 0.47 \\
\hline$D$ Minimum & 1.0 & 0.0012 & 0.00079 \\
$\vec{D}$ Minimum & 0.013 & 0.0012 & 0.00011 \\
\hline$D$ Median & 1.0 & 0.080 & 0.042 \\
$\vec{D}$ Median & 0.57 & 0.044 & 0.022 \\
\hline$D$ Mean & 1.17 & 0.15 & 0.059 \\
$\vec{D}$ Mean & 0.70 & 0.062 & 0.029 \\
\hline$D$ Std Deviation & 0.96 & 0.23 & 0.063 \\
$\vec{D}$ Std Deviation & 1.26 & 0.064 & 0.025 \\
\hline
\end{tabular}

Table 2: Recorded run-time complexity ratios of actual over potential run-time complexity using 10,794 year-long PASes. The table demonstrates the differences between traditional, non-time enforcing diameter $D$ and a time-constrained diameter $\vec{D}$. In average cases, our time-constrained diameter algorithm has approximate complexity $\frac{O(|V|+|A|)}{2}$ and is generally half the operational complexity of the non-time-enforcing diameter calculation. However, in the few instances where a PAS has multiple cycles, time-constrained diameter becomes more expensive. The PASes used for complexity analysis had at least one arc within the graph, thus explaining the smaller number of 10,794 compared with overall data set's 11,593 users.

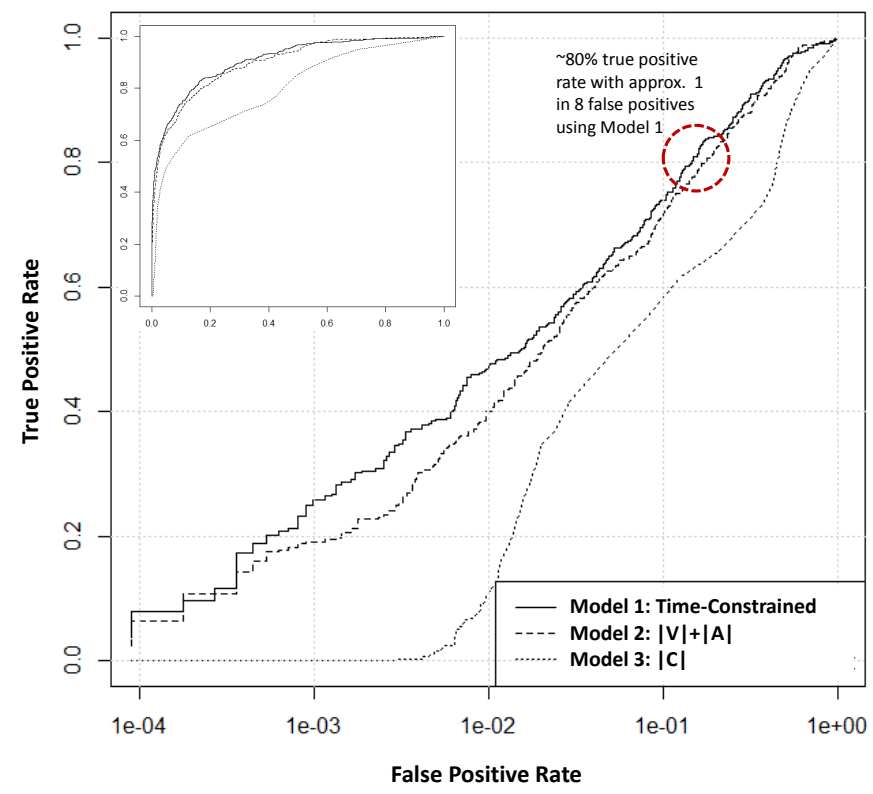

Figure 13: ROC curves comparing logistic regression classifiers for differentiating administrative users and general users based PAS feature sets. Model 1 has an area under the curve (AUC) of 0.907 , model 2 has an AUC of 0.897, and model 3 has an AUC of 0.798. The ROC curves are the averages from a 10 -fold cross validation. The inset figure shows a traditional ROC curve, while in the main figure the $\mathrm{x}$-axis is log-scale to help highlight the ROC curves at the lower false positive rates that matter the most. Model definitions are given in Section 4.1. 


\begin{tabular}{|c|c|c|c|}
\hline$\beta$ & Model 1 & Model 2 & Model 3 \\
\hline$\left|V_{u}\right|$ & $\begin{array}{c}0.035^{* * *} \\
(0.004)\end{array}$ & $\begin{array}{c}0.030^{* * *} \\
(0.002)\end{array}$ & $\begin{array}{l}\text { Not } \\
\text { Used }\end{array}$ \\
\hline$\overline{\left|A_{u}\right|}$ & $\begin{array}{c}-0.003^{* * *} \\
(0.001)\end{array}$ & $\begin{array}{c}0.002^{* * *} \\
(0.001)\end{array}$ & $\begin{array}{l}\text { Not } \\
\text { Used }\end{array}$ \\
\hline$\overline{\rho_{u}}$ & $\begin{array}{c}-23.357^{* * *} \\
(3.289)\end{array}$ & $\begin{array}{l}\text { Not } \\
\text { Used }\end{array}$ & $\begin{array}{l}\text { Not } \\
\text { Used }\end{array}$ \\
\hline $\overrightarrow{\vec{D}}_{u}$ & $\begin{array}{c}0.424^{* * *} \\
(0.084)\end{array}$ & $\begin{array}{l}\text { Not } \\
\text { Used }\end{array}$ & $\begin{array}{l}\text { Not } \\
\text { Used }\end{array}$ \\
\hline$\overline{\left|C_{u}\right|}$ & $\begin{array}{c}-0.015^{* * *} \\
(0.006)\end{array}$ & $\begin{array}{l}\text { Not } \\
\text { Used }\end{array}$ & $\begin{array}{c}0.035^{* * *} \\
(0.003)\end{array}$ \\
\hline$\overline{S_{u}}$ & $\begin{array}{c}1.486^{* * *} \\
(0.167)\end{array}$ & $\begin{array}{l}\text { Not } \\
\text { Used }\end{array}$ & $\begin{array}{l}\text { Not } \\
\text { Used }\end{array}$ \\
\hline$\overline{\text { Constant }}$ & $\begin{array}{c}-6.898^{* * *} \\
(0.337)\end{array}$ & $\begin{array}{c}-4.936^{* * *} \\
(0.096)\end{array}$ & $\begin{array}{c}-3.653^{* * *} \\
(0.060)\end{array}$ \\
\hline
\end{tabular}

Table 3: Logistic regression classifier details for differentiating administrative users from general users with results shown in Figure 13. For each of the models, the attribute coefficients $\beta$ are shown along with the standard error values in parenthesis under each $\beta$ value. Note that all coefficients are significant at the 0.01 level. 


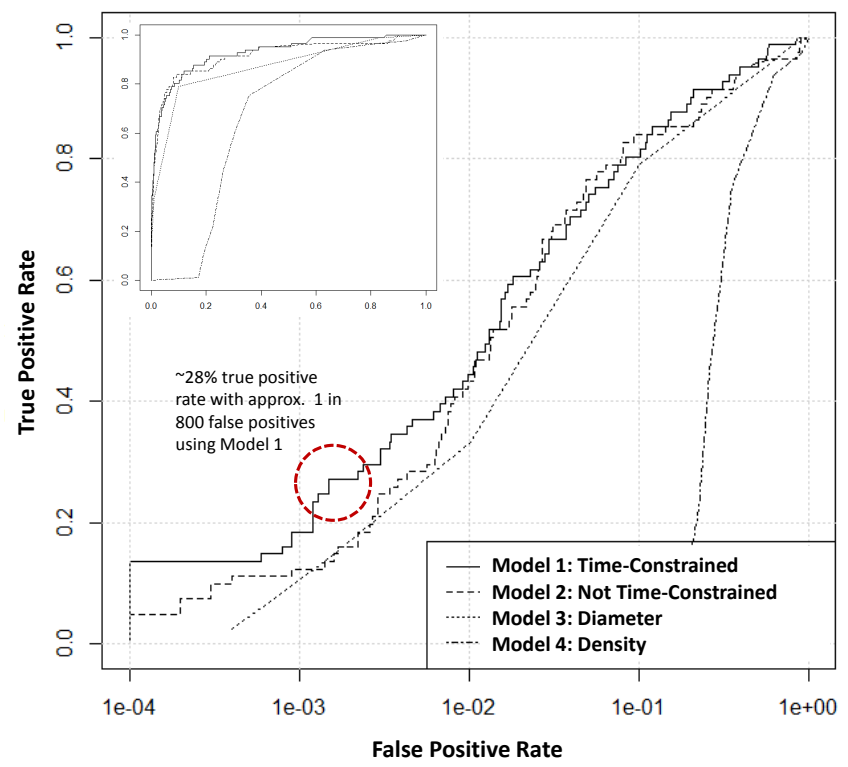

Figure 14: Averaged ROC curves comparing logistic regression classifiers for detecting compromised users. Model 1 has an area under the curve (AUC) of 0.927 , model 2 has an AUC of 0.915 , model 3 has an AUC of 0.870, and model 4 has an AUC of 0.665. The ROC curves are the averages from a 10 -fold cross validation. The inset figure shows a traditional ROC curve, while in the main figure the $\mathrm{x}$-axis is log-scale to help highlight the ROC curves at the lower false positive rates that matter the most. Model definitions are given in Section 4.2. 


\begin{tabular}{lcccc}
\hline \hline$\beta$ & Model 1 & Model 2 & Model 3 & Model 4 \\
\hline$\left|V_{u}\right|$ & $0.071^{* * *}$ & Not & Not & Not \\
& $(0.015)$ & Used & Used & Used \\
\hline$A_{u} \mid$ & $-0.019^{* * *}$ & Not & Not & Not \\
& $(0.006)$ & Used & Used & Used \\
\hline$\rho_{u}$ & $-10.210^{* * *}$ & $-20.843^{* * *}$ & Not & $-10.161^{* * *}$ \\
& $(3.519)$ & $(3.120)$ & Used & $(1.861)$ \\
$\vec{D}_{u}$ & $1.113^{* * *}$ & Not & Not & Not \\
& $(0.187)$ & Used & Used & Used \\
\hline$D_{u}$ & Not & $1.194^{* * *}$ & $1.902^{* * *}$ & Not \\
& Used & $(0.160)$ & $(0.121)$ & Used \\
\hline$S_{u \vec{P}}$ & $2.101^{* * *}$ & Not & Not & Not \\
& $(0.451)$ & Used & Used & Used \\
\hline$S_{u P}$ & Not & $1.528^{* * *}$ & Not & Not \\
& Used & $(0.262)$ & Used & Used \\
\hline$S_{u V T}$ & $-3.264^{* * *}$ & Not & Not & Not \\
& $(1.263)$ & Used & Used & Used \\
\hline Constant & $-7.914^{* * *}$ & $-6.608^{* * *}$ & $-7.495^{* * *}$ & $-4.052^{* * *}$ \\
& $(0.513)$ & $(0.348)$ & $(0.264)$ & $(0.150)$ \\
\hline \hline Note: & & & & $* * * \mathrm{p}<0.01$ \\
& & & &
\end{tabular}

Table 4: Logistic regression classifier details for predicting users that have their authentication credentials compromised with results shown in Figure 14. For each of the models, the attribute coefficients $\beta$ are shown along with the standard error values in parenthesis under each $\beta$ value. Note that all coefficients are significant at the 0.01 level. 


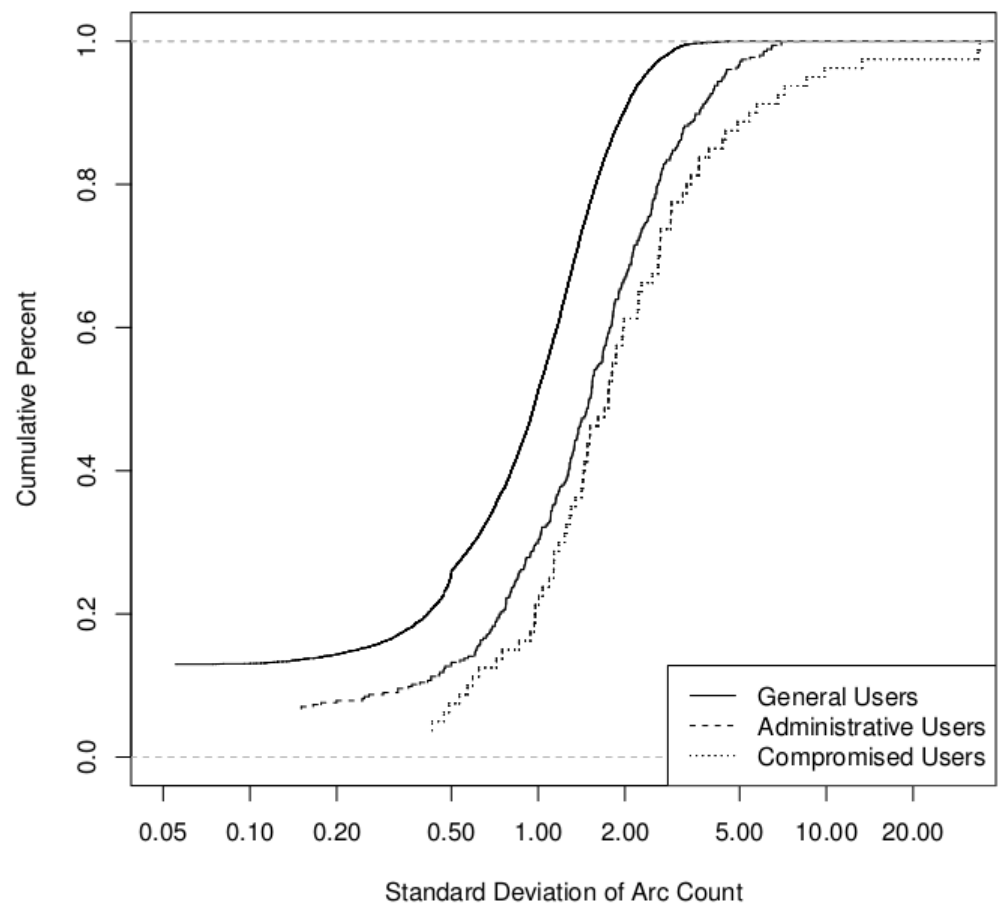

Figure 15: Cumulative distribution functions showing the differences in the standard deviation of PAS arc count over 2 hour window time series data labeled as general users, administrative users, and compromised users. This figure helps show the increased variance that exists in the PASes over time for administrative users and compromised users as compared with general users. 
Figure 17: Users $(U i \in U(B))$ and computers $(C i \in V(B))$ in a simplified BAG. The top graph shows a subset of a real system-level credential found to span a significant number of other systems within our 1 month data set. The lower graph shows that when we remove (or otherwise control) the access of user U1, the resulting graph shows a substantial increase in the number of connected component subgraphs. Understanding opportunities and changes of this type can be used to reduce the likelihood of credential stealing that allows a malicious actor to jump across discrete groupings of users. 